Notes on another geometry of harmony and proportion

PALABRAS CLAVE | PROPORCIÓN I GEOMETRÍA | ARMONÍA| ARPUITECTURA | ONDAS Y CUERDAS

KEYWORDS | PROPORTION | GEOMETRY | HARMONY | ARCHITECTURE I WAVES AND STRINCS

\section{| RESUMEN |}

El presente artículo expone los principales hallazgos del libro ACORDES ARPUITECTÓNICOS: OTRA GEOMETRÍA DE LA PROPORCIÓN Y LA ARMONÍA EN LA ARPUITECTURA, editado el año 2012. Mediante el estudio de nuevas teorías físicas es posible reconocer la unidad esencial y básica del conocimiento antiguo sobre la proporción y la armonía, base de la geometría y de los trazados reguladores de la arquitectura. En definitiva, una investigación que vincula los cambios en la física y su relación con la arquitectura y su carácter proyectual.

\section{ABSTRACT}

This paper presents the main findings of the book ARCHITECTURAL CHORDS: ANOTHER GEOMETRY OF

PROPORTION AND HARMONY IN ARCHITECTURE, published in 2012. Through the study of new physical theories it is possible to recognize the essential and basic unit of ancient knowledge about proportion and harmony, based on the geometry and the regulating lines of based on the geometry and the regulating lines of
architecture. In short, research that links physical changes and their relationship with architecture and its projective character

\title{
Anotaciones a otra geometría de la proporción y la armonía*
}

\author{
MARÍA LUISA LÓPEZ SARDÁ**. Universidad Alfonso X El Sabio, España·mllsarda@telefonica.net
}

Fecha de recepción 16/septiembre/2015 · Fecha de aceptación 12/diciembre/2015

T

al vez la definición más sugerente y sintética sobre el concepto de proporción, es la que da Platón en el diálogo TIMEO (1979) cuando dice: "Es imposible combinar dos cosas sin la existencia de una tercera. Es preciso que haya un vínculo que las una. No hay mejor vínculo que el que hace de sí mismo y de las cosas que une un todo único e idéntico".

En la disciplina de la arquitectura, Vitrubio desarrolló esta definición en tres líneas u opciones de entendimiento (1955). En primer lugar, como analogía o relación de semejanza entre partes o aspectos de objetos o sistemas diferentes. En segundo lugar, como correspondencia de medidas entre las partes y el todo. En tercer lugar, como relación de las partes y el todo con respecto a un módulo.

Y dentro del contexto del conocido paradigma newtoniano, el desarrollo, estudio e investigación de la proporción en la tratadística clásica de la arquitectura ha estado guiado y conducido por esta tríada vitrubiana, pero siempre en esa consideración del objeto arquitectónico en su aspecto espacial...

Paradigma que comenzó a fisurarse a comienzos del siglo XX, cuando aparecieron las teorías de la Relatividad General (T.R.G.) y de la Física Cuántica. La primera se ha convertido en el marco teórico de comprensión del universo a escala de estrellas y galaxias y la segunda en el marco teórico de comprensión del universo a escala subatómica.

La T.R.G. describe un continuo espacio tiempo de cuatro dimensiones cuyo marco geométrico depende del contenido de materia y energía y donde ya no existe una marco de referencia absoluto para interpretar un fenómeno sino que dependerá del marco de referencia de cada observador.

El presente artículo forma parte del libro ACORDES ARQUITECTÓNICOS: OTRA GEOMETRÍA DE LA PROPORCIÓN Y LA ARMONÍA EN LA ARQUITECTURA, publicado por la autora el año 2012.

** Arquitecto por la Escuela Técnica Superior de Arquitectura de Madrid (ETSAM), España (1972), especialidad de Urbanismo. Doctor-Arquitecto por la Universidad Alfonso X El Sabio de Madrid, España (2011). Ha trabajado en el estudio de los arquitectos Francisco Javier Saenz de Oíza, Rafael Moneo y Carlos Ferrán. Desde 1976 tiene estudio profesional abierto en Madrid: www.acordesarquitectonicos.com. Ha publicado los libros SANTA MARía DE Veruela. Armonía en el trazado geométrico. (l' y 2' parte). E. Electa 1994 y 1996; Arquitectura de representación: España de Oriente a Occidente. M AA EE, MOPTMA, FUCOAM. 1995; Acordes Arquitectónicos: otra geometría de la proporción y la armonía en la arquitectura. Ed. Fisuras 2012. Desde el año 2000, ha sido profesora de Proyectos 2 ( $4^{\circ}$ curso), coordinadora de la asignatura (2002-2006) y tutora de Proyectos Fin de Carrera del Departamento de Arquitectura de la Universidad Alfonso X El Sabio en Madrid, España. Profesora invitada en la Universidad de Woodbury en Los Angeles, Estados Unidos, en el verano 2008 

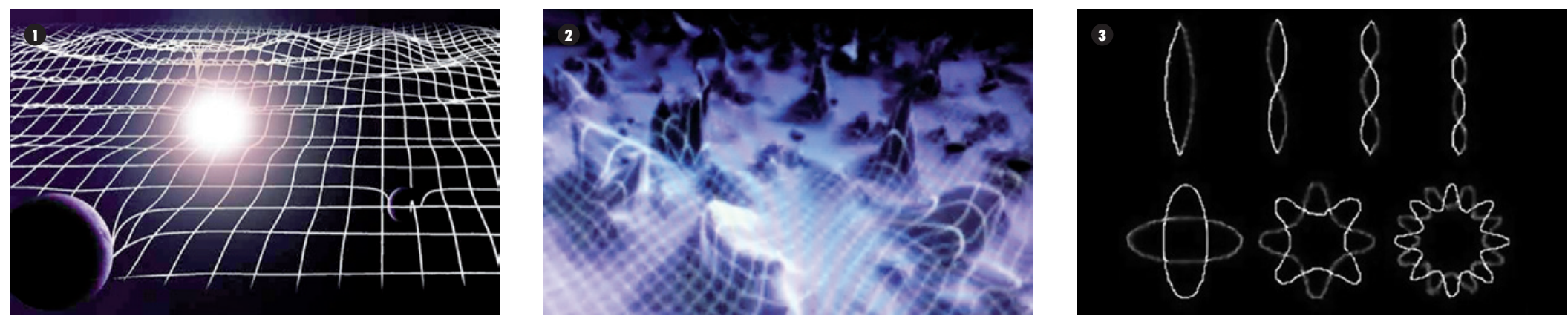

Esta interrelación entre espacio tiempo y materia queda expresada en la frase del físico J. Wheeler: "La materia le dice al espacio cómo ha de curvarse. El espacio le dice a la materia cómo ha de moverse" (Kaku, 2008) (Figura 1).

La Mecánica Cuántica define que las partículas elementales son patrones dinámicos que tienen un cierto aspecto espacial en cuanto objeto con una cierta masa y un aspecto temporal como proceso que encierra una energía... Esto es, la conocida "dualidad onda-partícula" que Neils Bohr definió como principio de complementariedad en la que las palabras, onda y partícula, son consideradas como dos descripciones de la misma realidad (Green, 2007) (FIGURA 2).

Y estos dos pilares en los que se apoya la física actual, parecen tener sus diferencias puesto que la estructura curvada del espacio tiempo de la Relatividad General queda en entredicho por las continuas y constantes fluctuaciones del mundo cuántico. De las múltiples teorías que han surgido en los últimos cincuenta años para relacionar, podríamos decir, armonizar, estos dos marcos teóricos de referencia, está la llamada teoría de cuerdas, o teoría de Branas o teoría M.

La teoría de cuerdas plantea que si pudiéramos examinar con mayor precisión esas partículas elementales, descubriríamos que ninguna es un punto sino que cada partícula contiene un filamento que oscila y vibra y que los físicos han llamado cuerda. Esta teoría sugiere que el paisaje microscópico está cubierto por diminutas cuerdas cuyos modelos de vibración definen la evolución del universo. Cada una de estas cuerdas puede ejecutar una gran variedad de modelos de vibración diferentes conocidos por una sola cuerda y todas son idénticas. Las diferencias entre partículas son los diferentes modelos resonantes de vibración... es decir, los diferentes modelos de vibración de cada cuerda definen la evolución de la materia en el universo... (FIGURA 3).

Y Michio Kaku (2006) escribe: “De manera que las leyes de la física, cuidadosamente construidas después de miles de años de investigación, no son más que las leyes de la armonía para cuerdas y membranas".

Y ahora, a comienzos del siglo XXI, el flujo de ideas provenientes de la física plantea un universo como un complejo entramado de eventos. Un universo donde las partículas materiales son abstracciones cuyas propiedades se definen y observan a través de sus interacciones con otros sistemas, donde la materia no existe con certeza sino que más bien, muestra "tendencia a existir" y los sucesos atómicos no ocurren en tiempos definidos, sino que muestran "tendencias a ocurrir" y estas tendencias se expresan como probabilidades (Capra, 1992). Un universo donde los objetos de la física clásica se disuelven en patrones ondulatorios de probabilidades, pero no de cosas, sino de probabilidades de interconexiones. Un universo donde la partícula es manifestación del campo, campo de fuerza por resonancias y cada partícula está formada como entidad, como región del espacio con leyes y estructura propia... Un universo no ya como una máquina según el paradigma newtoniano, compuesto de multitud de objetos, sino un todo dinámico e indivisible cuyas partes están íntimamente ligadas y donde la presencia del espectador modifica el experimento...

Este importantísimo desarrollo del conocimiento científico forma parte del desarrollo actual y por tanto, tiene resonancias en el arte en general y en la arquitectura en particular puesto que ambas están inmersas en la comprensión del universo, en la forma de ver el mundo que la sociedad o el conocimiento científico tienen en cada época.

Mencionaría dos pintores y cuatro arquitectos del siglo XX, todos muy diferentes entre sí formalmente pero muy próximos en la intuición de esas nuevas realidades que ponen de manifiesto esa resonancia entre arte y ciencia.

En primer lugar a Escher. Destacaría la serie de litografías Ilamadas METAMORFOsis, como expresión de ese continuo fluir de la vida. Comenzando con una palabra Metamorfosis, esta se desarrolla y organiza y en el proceso, pequeñísimos cambios en el valor de sus parámetros devienen en nuevas morfologías.

Victor Vasarely. Un estudioso de las teorías de la relatividad y de la mecánica cuántica como él mismo expresa. Destacaría los cuadros VEGA del año 1957 o BIADAN del 1959: modificaciones tensionales de expansión y contracción de una 
retícula cartesiana, diferentes expresiones de cómo la materia deforma la tela del espacio tiempo.

Toyo Ito y la Mediateca de Sendai. Define las plantas como diagramas de acciones, como representaciones gráficas en la variación de las acciones. Define las secciones como chimeneas de flujos y fuerzas que se densifican y distancian a intervalos variables..

\section{El Parque de la Relajación en Torrevieja} (Alicante) del año 2002 entendiendo la arquitectura como generación de remolinos en las corrientes naturales o artificiales, como dunas en la playa formalizadas por las espirales de las conchas.

Peter Eisenmann y la Ciudad de la Cultura en Santiago de Compostela. En este caso, arquitectura entendida como superposición de capas de información y donde una malla generada por ordenador a partir de dichas tramas configura una superficie curvada que cae sobre la colina...

\section{Frank Gehry y el Museo Guggenheim en}

Bilbao que anticipó una visión de la arquitectura como amalgama de volúmenes interconectados delimitados por sinuosas curvas de piedra, cristal y titanio..

Zaha Hadid. Destacaría el proyecto para la Gran Mezquita de Estrasburgo del año 2000 que expresa ese entendimiento del proyecto como campos de flujos y fuerzas que se enrollan y se mueven unos sobre otros y se convierten en paisajes solapados. La distinción que junto a su socio Patrik Schumacher hacen entre el concepto del espacio del movimiento moderno y el concepto de campo del movimiento paramétrico...

En este contexto se ha desarrollado esta investigación. Preguntas que han encaminado el proceso y formulado una hipótesis:

¿Qué sucedería si volvemos al origen pitagórico y estudiamos la proporción y la armonía como un proceso de oscilación y vibración de una cuerda?

Cuerda como resonancia de la teoría de cuerdas, como primer y único elemento cuyo sistema de vibración define la diferenciación de la materia en el universo.

¿Qué sucedería si conceptos eternos de la arquitectura en su definición del espacio, como proporción, geometría y armonía, los estudiamos de nuevo a la luz de las nuevas teorías físicas?

Proporción, geometría y armonía como resonancia de esa dualidad "onda-partícula" asociada al comportamiento de la materia.

El proceso comenzó dejando aparcados lo que se sabe y enseña sobre proporción y armonía y volviendo de nuevo al origen, al estudio de la oscilación de una cuerda de longitud unidad...

El impresionante descubrimiento de Pitágoras fue que el tono musical depende de la longitud de una cuerda que vibra. Esto es, las diferencias cualitativas del sonido se podían traducir en diferencias cuantitativas. Una cualidad podía representarse por una cantidad y dos verbos, aparentemente opuestos como cuantificar y cualificar quedaron relacionados mediante el número. El Número vinculó Cualidad y Cantidad...
Pero han pasado unos 2.500 años desde que Pitágoras comenzó haciendo vibrar una cuerda y hallando sus armónicos. Hoy a estos diferentes modelos de vibración de una cuerda se les llama ondas estacionarias. Esta distancia temporal ha permitido que la sensibilidad y el afinamiento del sentido del oído de los antiguos capaces de establecer los sonidos como relaciones sencillas de números enteros y que sentaron las bases de la música, ha sido por un lado corroborado, y por otro sustituido por el control científico de los armónicos definidos por el número de vibraciones por unidad de tiempo.

Hoy el tema es más complejo [1]. El desarrollo científico desde finales del XIX y principios del XX dio el gran salto al considerar la materia desde el punto de vista ondulatorio y hoy el estudio de ondas forma parte de las disciplinas de física y matemáticas ${ }^{[2]}$ y dentro de esta última, del área denominada Análisis Armónico o Análisis de Fourier ${ }^{[3]}$. Así por ejemplo, cuando la onda que se produce, varía sinusoidalmente con el tiempo, recibe el nombre de armónica o sinusoida| ${ }^{[4]}$.

Desde el punto de vista físico una onda es una forma de propagación acompañada de una transmisión de energía en un medio material que no supone desplazamiento de materia. Y una onda estacionaria es la interferencia de dos movimientos ondulatorios armónicos de la misma longitud de onda; es decir, una interferencia entre una onda incidente que se

[1] Es interesante aclarar la distinción que hace R. Penrose (2007) sobre el cálculo infinitesimal. Dice que este está construido sobre dos ingredientes básicos: la diferenciación y la integración. El cálculo diferencial está relacionado con velocidades y aceleraciones, con pendientes y curvatura de curvas y superficies y cosas similares. Son tasa de cambio de las cosas. El cálculo integral está relacionado con áreas y volúmenes, con centros de gravedad y con muchas otras cosas de esa misma naturaleza.

[2] Matemáticamente, a los distintos modos de vibración de una cuerda, se les llama los armónicos superiores de una única cantidad compleja eix $=\cos x+i \operatorname{sen} x$, para un período $L=2 \pi$, de manera que e $i 2 \pi x L$, $\operatorname{sen}(2 \pi n x / L)$, $\cos (2 \pi n x / L)$, donde $n=2,3,4 \ldots$ Penrose, R. El CAMINO A LA REALIDAD. Ídem. anterior.

[3] Este sistema de análisis consiste en el estudio de formas de ondas complejas mediante su descomposición, O superposición de un número suficientemente grande, de ondas básicas o armónicos.

[4] Se define la elongación como el valor de la perturbación en un determinado punto o instante siendo su valor máximo la Amplitud. Cuando la elongación varía sinusoidalmente con el tiempo según la ley y $=$ A.sen y = elongación, $A=$ amplitud,$t=$ tiempo, $\omega=$ pulsación, recibe el nombre de onda armónica. Véase $L A$ EnCiclopedia El País. Tomo N 15. (2003) Salvat Editores S.A. 
4 y 5 . Dibujo de María Luisa López Sardá.

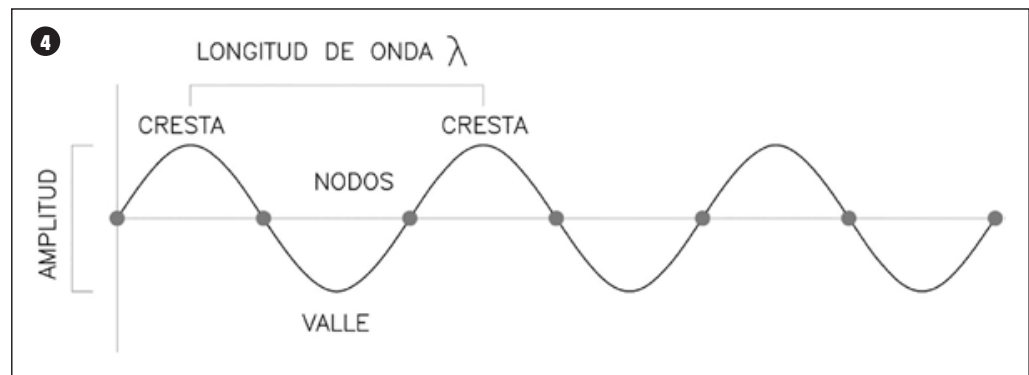

mueve de izquierda a derecha y otra onda reflejada que se mueve de derecha a izquierda, ambas de la misma amplitud y longitud de onda.

Y esta onda transversal se caracteriza por su velocidad ( $\mathrm{m} / \mathrm{seg}$ ), su amplitud A (distancia entre la máxima cresta y el máximo valle), su longitud de onda (distancia entre crestas o valles) y su frecuencia $f$ (definido por el cociente entre su velocidad y su longitud de onda o número de crestas que pasan por un punto definido en un segundo) medido en herzios, todas ellas afectadas por la propia densidad lineal de la cuerda.

Para nuestro estudio, el interés radica en su representación geométrica. Tomemos dos ejes de coordenadas donde el eje " $y$ " define la amplitud A y el eje " $x$ " la longitud de onda, porque la frecuencia, geométricamente, quedará definida por el número de nodos intermedios. Conviene aclarar que la "forma de la onda" es la instantánea que congela las posiciones de las partículas en un momento dado (FIGURA 4).

Para una cuerda, hay ciertas frecuencias que se llaman frecuencias de resonancia: la más baja es la frecuencia fundamental y las demás son múltiplos de ella. En nuestro caso, la frecuencia más baja en una cuerda de longitud $L$ es la que corresponde a $n=1$ y corresponde a la máxima distancia entre nodos (FIGURA 5).

Tomemos un medio elástico como una cuerda, definamos su modo de frecuencia y sus armónicos. Llamemos a los dos extremos fijos de la cuerda 1/1 y 2/1 y los nodos intermedios de vibración nula han quedado calificados. En definitiva, la división de una cuerda en dos, tres, cuatro, etc... partes iguales. En el segundo armónico surge el nodo intermedio $3 / 2^{[5]}$, en el tercero, surgen $4 / 3$ y $5 / 3^{[6]}$, en el cuarto $5 / 4,3 / 2$ y $7 / 4^{[7]}$, y así sucesivamente para el modo de vibración séxtuple, óctuple, etc. (FIGURA $6)$.

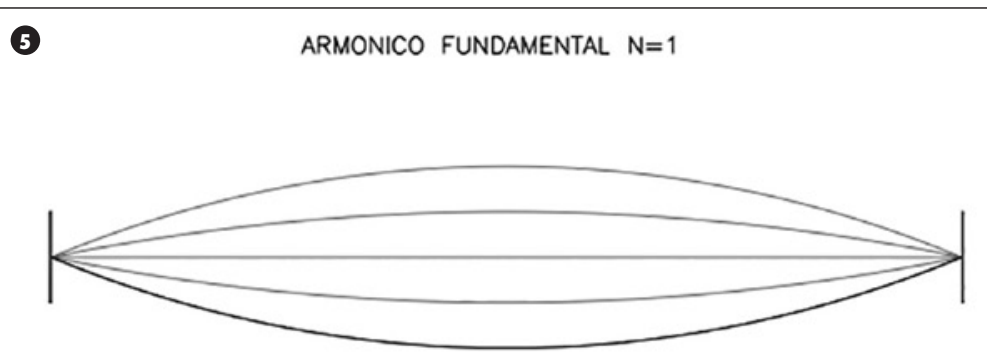

SEGUNDO ARMONICO $\mathrm{N}=2$

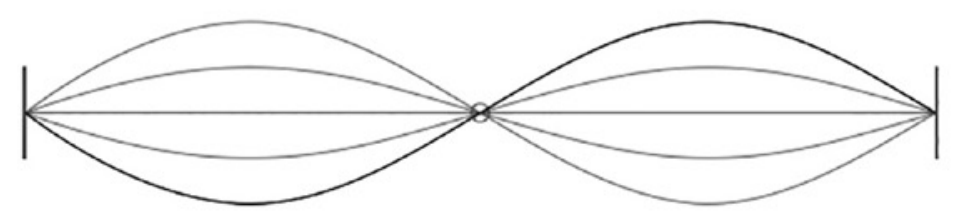

TERCER ARMONICO $\mathrm{N}=3$

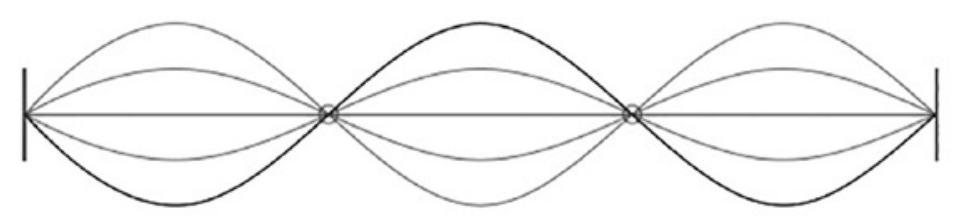

CUARTO ARMONICO $\mathrm{N}=4$

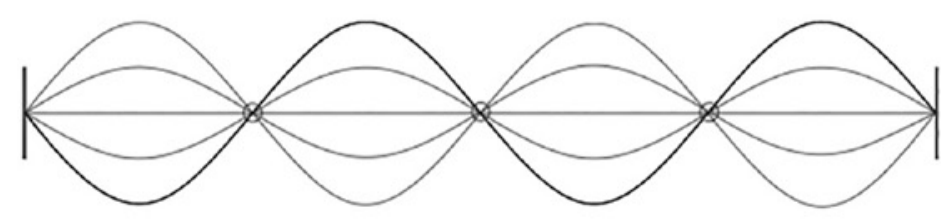

LONGITUD DE CUERDA L 


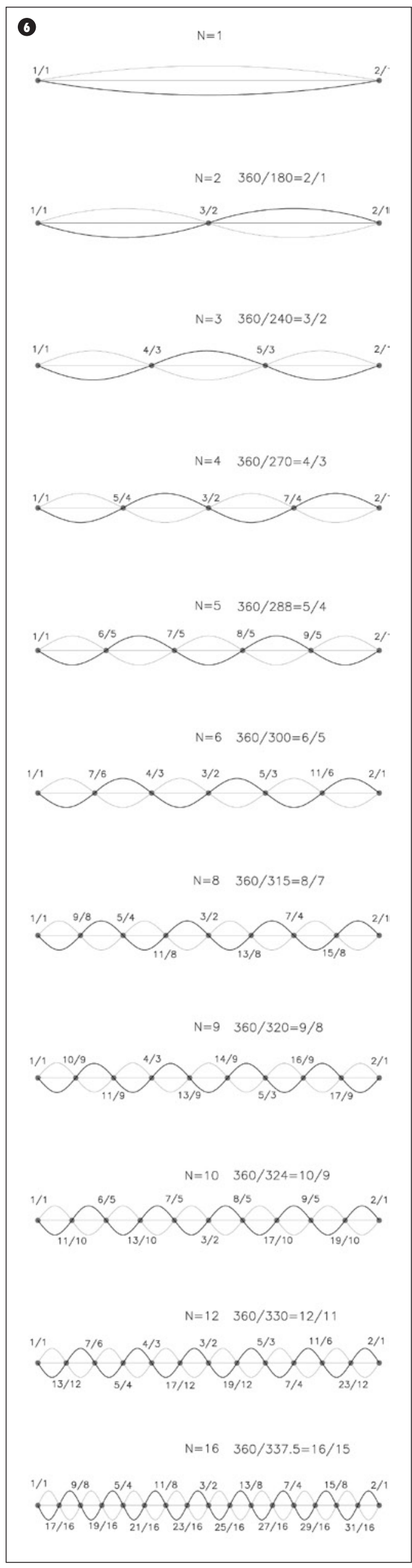

6 y 7. Dibujo de María Luisa López Sardá.

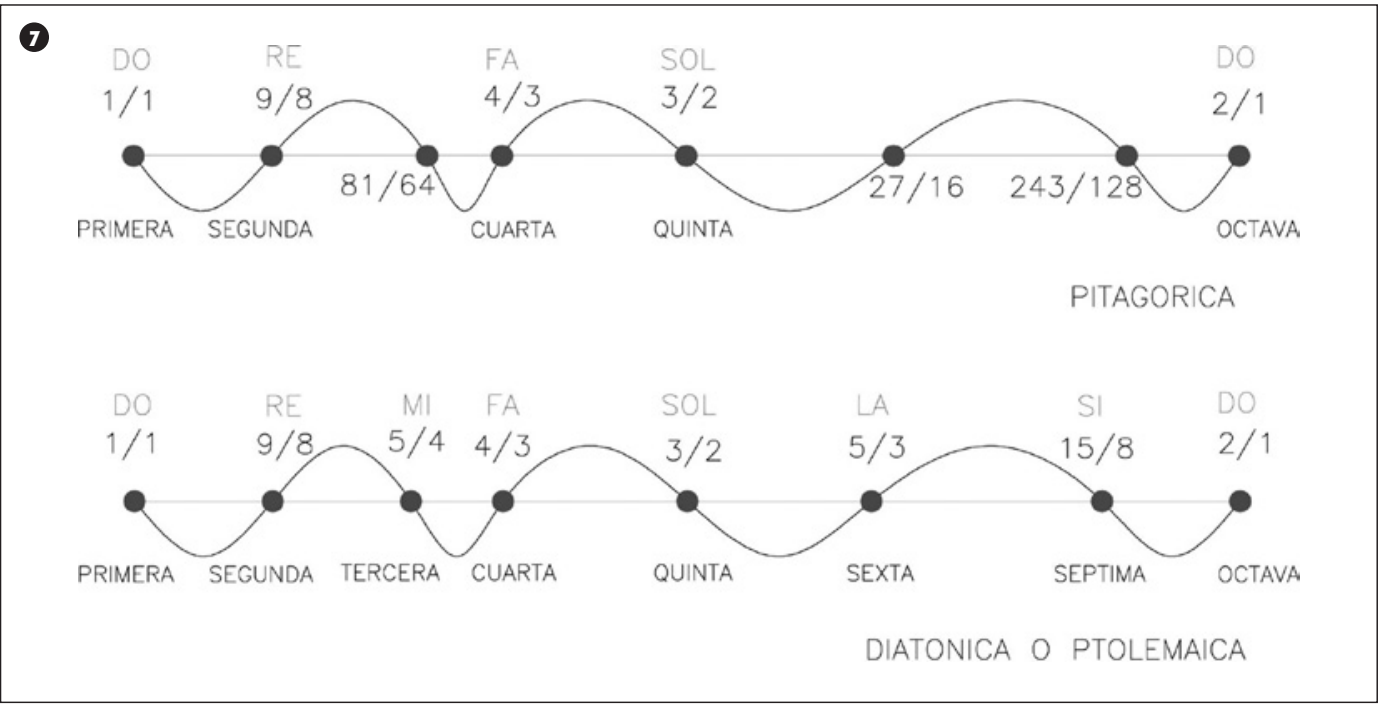

Lo interesante es que comienzan a aparecer una serie de relaciones sencillas de números enteros que traen a la memoria las relaciones básicas que los griegos utilizaron para definir su gama musical. De esta manera, si $1 / 1$ define el Unísono y 2/1 define la Octava, el primer nodo del segundo armónico 3/2 define la Quinta o nota Sol. En el modo de vibración triple, el nodo intermedio 4/3 define la Cuarta o nota Fa y la relación 5/3 define la Sexta Mayor o nota La. En el modo de vibración cuádruple tenemos el nodo 5/4 que define la Tercera Mayor o nota $\mathrm{Mi}$, el 3/2 ya mencionado y la relación 7/4 o aproximación por exceso a En el modo de vibración quíntuple, surge la relación 6/5 o Tercera menor, la relación 7/5 o aproximación a 2 , la relación 8/5 o Sexta menor pero también, relación de dos números consecutivos de la serie Fibonacci, y por último, la relación 9/5 ...

Es decir, por el solo hecho de "nombrar" los extremos de la cuerda, los nodos intermedios han quedado calificados y el resultado es una serie de relaciones sencillas de números enteros que hacen referencia a la octava musical (FIGURA 7).

Si tomamos esta longitud de la cuerda y sus distintos modos de vibración armónica ya definidos y unimos sus extremos, podemos convertirla en la longitud de una circunferencia en cada caso, pautada o marcada por los nodos intermedios. Lógicamente, si unimos los puntos de vibración nula o nodos, obtenemos la serie de polígonos elementales donde cada vértice del polígono está definido por una relación de dos números enteros que corresponde al nodo de la cuerda (FIGURA 8).

En el segundo armónico, al unir los nodos, aparece el diámetro de la circunferencia. Con este armónico, tenemos el círculo y el diámetro y con ello entramos en el sistema geométrico de definir las proporciones o medias más usuales: la aritmética, la geométrica y la armónica.

El tercer armónico genera el triángulo equilátero con sus vértices "calificados".

El cuarto armónico genera el cuadrado girado $45^{\circ}$. En el modo de vibración quíntuple, el pentágono, en el modo séxtuple, el hexágono y así sucesivamente.

En nuestra cultura, se considera que música y geometría son disciplinas independientes pero esta idea es equívoca puesto que con esta sencilla operación, la Geometría ha quedado 
8. Dibujo de María Luisa López Sardá.

vinculada con las proporciones musicales, es decir, con la Armonía. $\bigcirc$ lo que es lo mismo, el objeto geométrico, el polígono, ha quedado vinculado a la onda.

Platón, en el diálogo TiMEO (1979), describe una cosmología desde los principios fundamentales hasta la creación de los animales. Cuando pasa a describir la formación y disposición de los cuerpos elementales: fuego, agua, aire y tierra, utiliza un lenguaje geométrico que no es usual. Tres tipos de triángulos son el origen de estos cuerpos: escuadra, cartabón y triángulo equilátero.

Sin discutirle la belleza de esta primera tríada de triángulos, si podemos examinar algunos triángulos más que nos ofrecen los polígonos elementales ${ }^{[8]}$.

Así, en el cuarto armónico, el cuadrado resultante de unir sus nodos está compuesto de dos escuadras o primer triángulo platónico (FIGURA 9).

En el quinto armónico, el pentágono ofrece dos triángulos isósceles que generan la misma construcción geométrica ${ }^{[9]}$ (FIGURA 10).

En el sexto armónico, el hexágono contiene dos de los triángulos de la tríada platónica, el cartabón y el equilátero que generan dos construcciones diferentes (FIGURA 11).

En el octavo armónico, el octógono ofrece tres triángulos. Además de la escuadra, el isósceles de $45^{\circ} / 67,5^{\circ} / 67,5^{\circ}$ y la escuadra $22,5^{\circ} / 67,5^{\circ} / 90^{\circ}[10]$ que generan tres diferentes construcciones geométricas (FIGURA 12).

Por último, el duodécimo armónico dodecágono ofrece cinco triángulos que de nuevo, generan cinco construcciones geométricas diferentes. Contiene además de la tríada platónica compuesta de escuadra, cartabón y equilátero, un isósceles de $30^{\circ} / 75^{\circ} / 75^{\circ}$ y un escaleno de $45^{\circ} / 60^{\circ} / 75^{\circ}[1]$ (FIGURA 13).

[8] "Por esto debemos escoger el más bello entre esta multitud de triángulos si queremos empezar convenientemente" (Platón, 1979).

[9] El isósceles de $36^{\circ} / 36^{\circ} / 108^{\circ}$, como gnomon del segundo: si tomamos la base como valor unidad, sus otros dos catetos toman el valor de $1 /$ phi. El segundo isósceles de $36^{\circ} / 72^{\circ} / 72^{\circ}$ : si tomamos la base como valor unidad, sus otros dos catetos toman el valor de phi.

[10] En este triángulo isósceles si tomamos la base como valor unidad, su altura es $(1+\sqrt{ } 2)=\theta$

[11] En el isósceles de $30 / 75^{\circ} / 75^{\circ}$ si tomamos la base como valor unidad, su altura es $(2+\sqrt{ } 3)$. El escaleno de $45^{\circ} / 60^{\circ} / 75^{\circ}$ está formado por la escuadra y el cartabón.
8

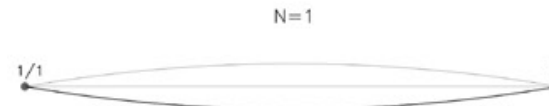
$2 / 1$
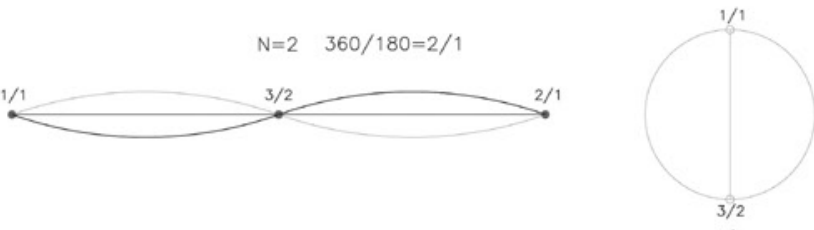

$N=3 \quad 360 / 240=3 / 2$
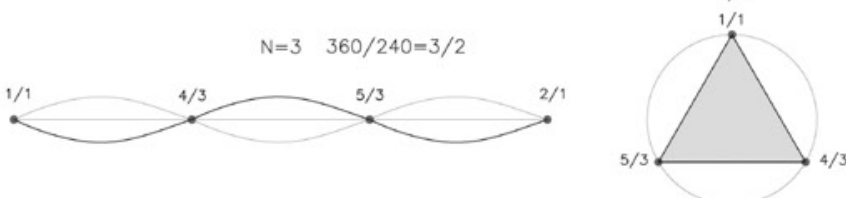

$=4 \quad 360 / 270=4 / 3$
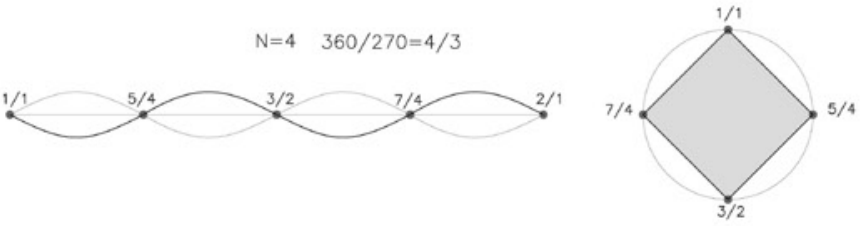

$N=5 \quad 360 / 288=5 / 4$
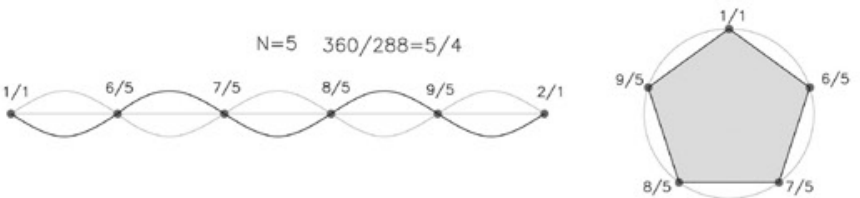

$N=6 \quad 360 / 300=6 / 5$
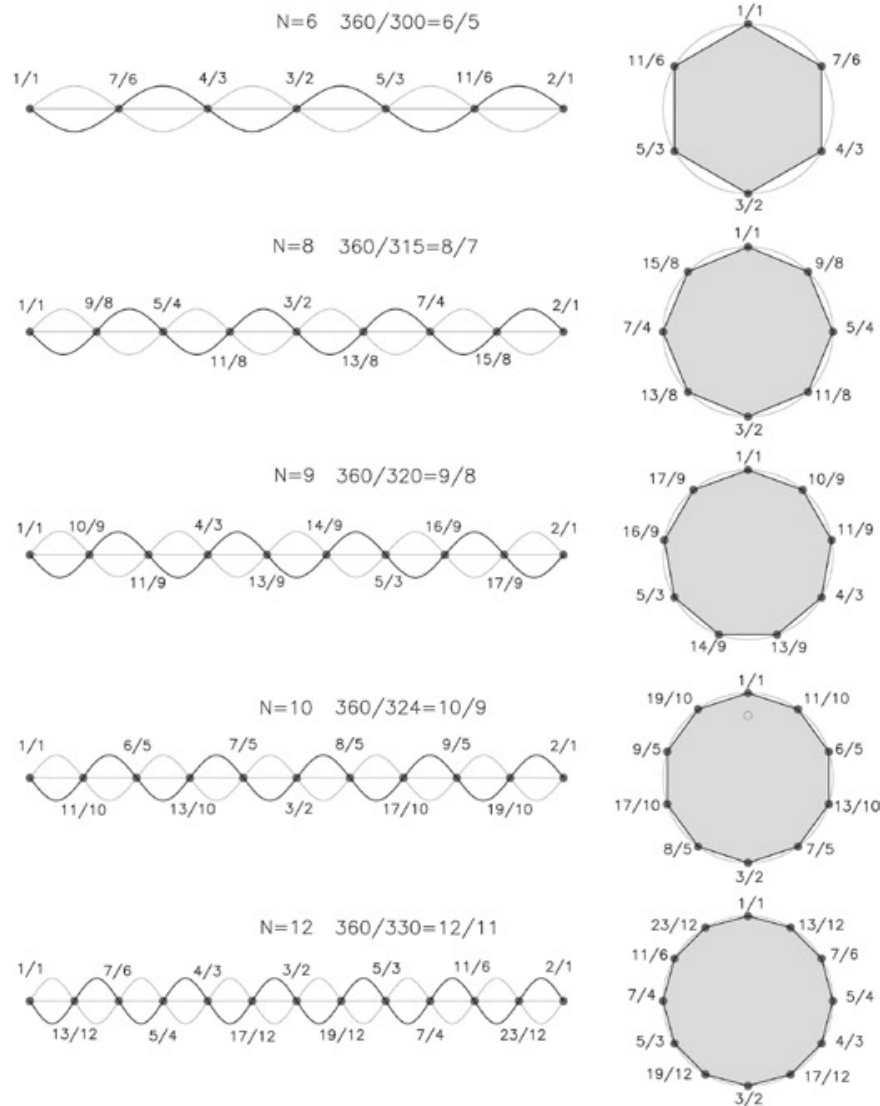

$\mathrm{N}=16 \quad 360 / 337.5=16 / 15$
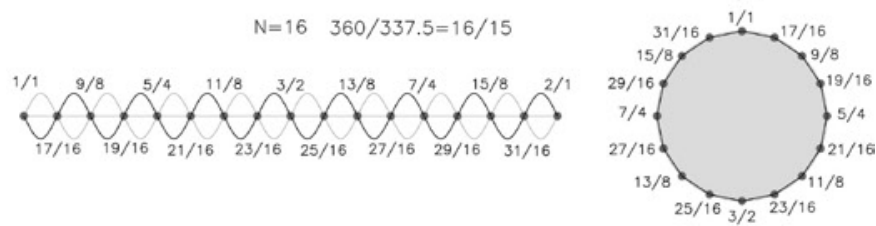
9, 10, 11 , 12 y 13. Dibujo de María Luisa López Sardá.

(

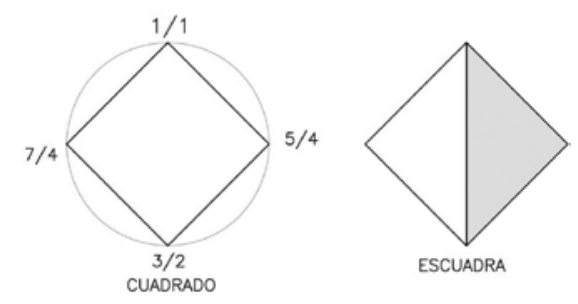

(1)
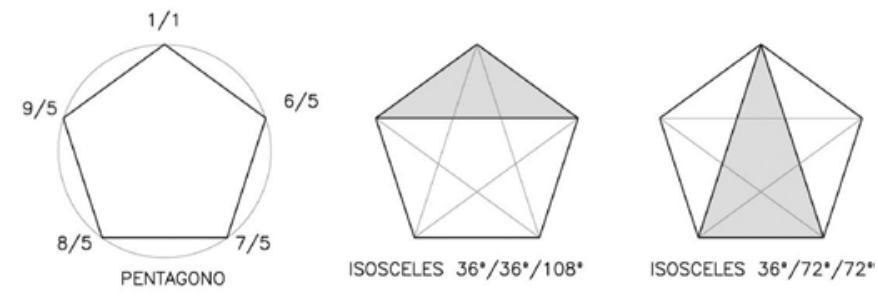

(1)
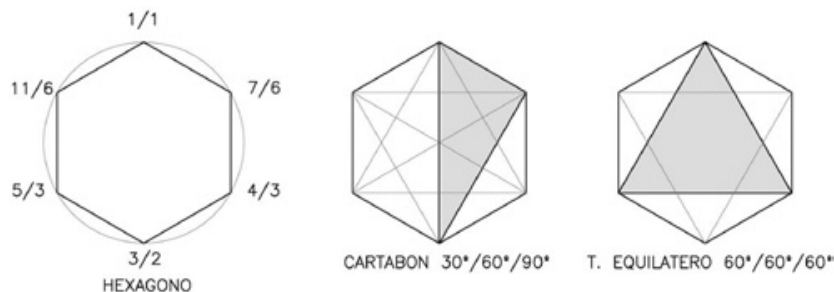

(1)
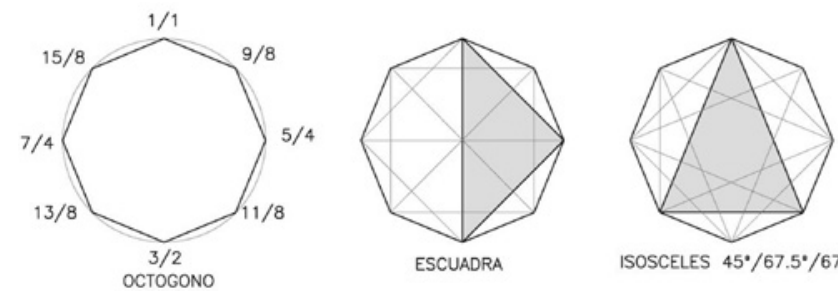

ISOSCELES $45^{\circ} / 67.5^{\circ} / 67.5^{*}$

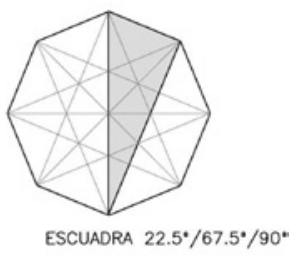

(B)
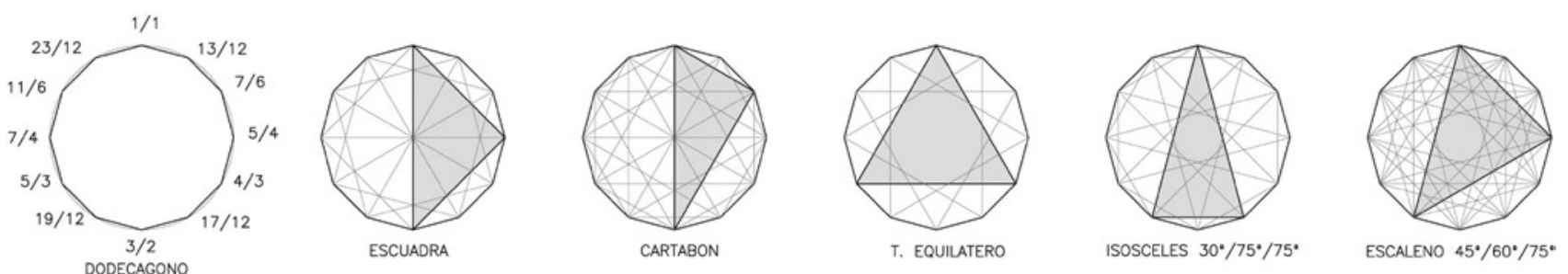
14, 15 y 16. Dibujo de María Luisa López Sardá.

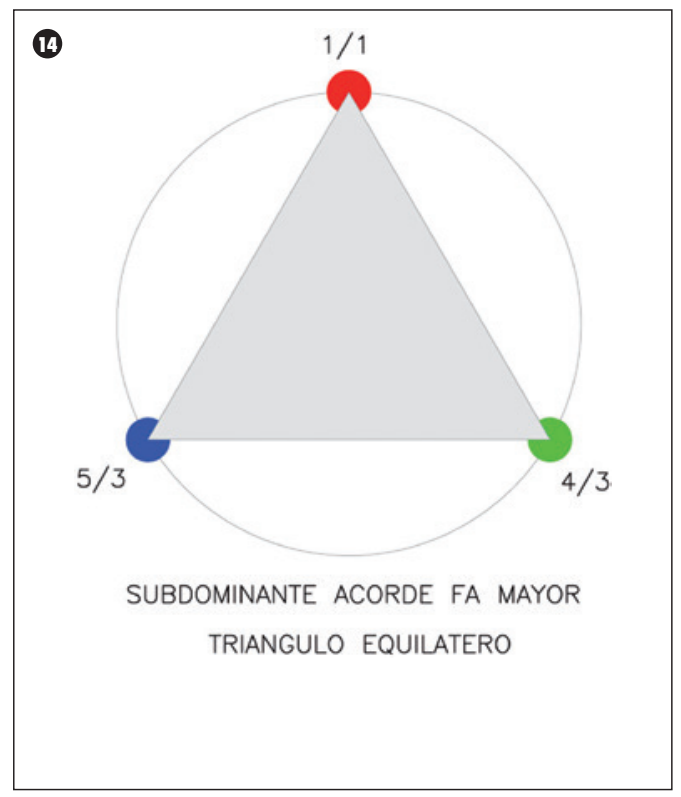

Pero no olvidemos que cada triángulo forma parte de alguno de los polígonos regulares y que cada polígono está inscrito en la longitud de una circunferencia que es la longitud de la cuerda en su modo de oscilación, pautada por los nodos calificados...

Esto permite afirmar la correspondencia entre triángulos y acordes musicales.

En el tercer armónico, el equilátero surgido al unir por líneas rectas los nodos intermedios tiene sus vértices en 1/1, 4/3 y 5/3. El Acorde mayor de Fa o Subdominante está definido por las notas $\mathrm{Do}(1 / 1), \mathrm{Fa}(4 / 3), \mathrm{La}(5 / 3)$, luego puede plantearse que el triángulo equilátero, geométricamente, defina este acorde musical (FIGURA 14).

En el cuarto armónico, la longitud de la circunferencia está pautada por los nodos $1 / 1$, 5/4, 3/2 y 7/4. El Acorde mayor de Do o Tónica está definido por las notas Do(1/1), Mi(5/4), Sol(3/2).

En el TIMEO, Platón define este isósceles como el primero: "origen que atribuimos al fuego y a los
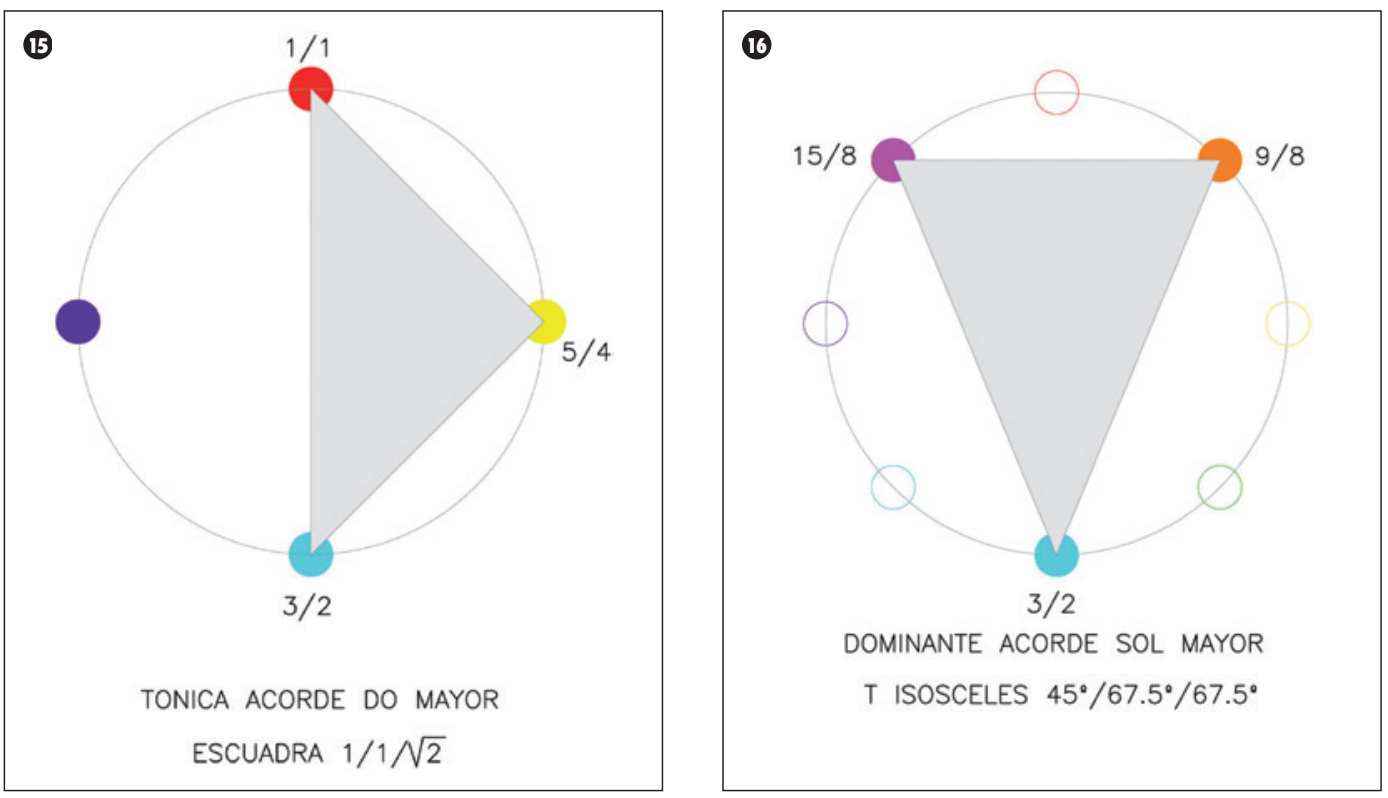

otros tres cuerpos obedeciendo a la necesidad" (1979). Luego tiene cierta verosimilitud que, geométricamente, la escuadra defina este acorde musical (FIGURA 15).

Es decir, de los tres acordes básicos de la música, Tónica, Dominante y Subdominante, dos de ellos: la Subdominante y la Tónica corresponden a dos de los triángulos que Platón en el TIMEO considera esenciales para la creación de los elementos del Mundo.

Para hallar el tercer, Acorde mayor de Sol o Dominante, hay que llegar al octavo armónico u octógono. El isósceles de $45^{\circ} / 67,5^{\circ} / 67,5^{\circ}$ con los vértices en las posiciones $9 / 8,3 / 2$ y 15/8, correspondientes en notación musical a las notas $\mathrm{Re}$, Sol y Si de la escala diatónica, puede definir este Acorde mayor básico (Figura 16).
Pero este mismo triángulo, con los vértices en las posiciones $5 / 4,13 / 8$ y $15 / 8$ puede corresponder al Acorde mayor de $\mathrm{Mi}$ : Mi(5/4), Sol\#(13/8), Si(15/8) ${ }^{[12]}$ (FIGURA 17).

Para el estudio de otros Acordes mayores, hay que examinar el armónico duodécimo o dodecágono. La posición de los vértices del triángulo isósceles de $30 / 75^{\circ} / 75^{\circ}$, en los nodos $13 / 12,5 / 4$ y $5 / 3$, puede corresponder al Acorde mayor de La: Do\#(13/12), Mi(5/4), La(5/3) ${ }^{[13]}$ (FIGURA 18).

Puede ser interesante examinar el tercer triángulo platónico: el cartabón. En el mismo dodecágono, o en el hexágono, este triángulo con los vértices en las posiciones $1 / 1,7 / 6$ y $3 / 2$ puede corresponder al Acorde menor de Do: Do(1/1), Mi bemol(7/6) [14], Sol(3/2). Tiene

[12] Las notas sostenidas suelen ser más próximas a la siguiente nota mientras que los bemoles suelen ser más próximos a la nota anterior; por ellos podemos considerar que el valor 13/8 funciona como un Sol\#.

[13] El mismo criterio anterior para considerar el valor 13/12 como un Do\#; así, la relación 13/12 contenida entre 1/1 y 9/8 es más próxima a 9/8.

[14] De nuevo, el mismo criterio; podemos considerar el valor 7/6 como un Mib. 
sentido que el Acorde mayor de Do corresponda a la escuadra y su Acorde menor, al cartabón (FIGURA 19).

Esto es, el examen de las correspondencias entre triángulos constitutivos de los polígonos y los acordes musicales de la escala diatónica, permite afirmar que:

- el octógono mediante determinadas posiciones de la escuadra y del isósceles de $45^{\circ}$ presenta correspondencias con los acordes mayores de las notas Do, Mi y Sol.

- el dodecágono mediante determinadas posiciones de la escuadra, del equilátero y del isósceles de $30^{\circ}$ presenta correspondencias con los acordes mayores de las notas Do, Fa y La.

Estas comprobaciones permite que sigamos el discurso y podamos afirmar que: los triángulos representan en geometría lo que los acordes en música y por ello, la Geometría ha quedado vinculada a la Música mediante la Armonía.

Vamos a estudiar esa "otra" geometría de la proporción y la armonía mediante el dodecágono. Este polígono por su sencillez, es un medio idóneo para entender todo el proceso, actúa como el abecedario para la construcción de las sílabas que se convierten en palabras, luego en frases y forman un lenguaje y contiene en número suficiente de triángulos para ver la gran riqueza de soluciones y las posibilidades contenidas en la geometría de ondas de un polígono de doce lados (FIGURA 20).

Recordemos que el dodecágono es el primer polígono que puede generarse por los tres triángulos platónicos además del isósceles de $30^{\circ}$ y el escaleno de $45^{\circ} / 60 / 75^{\circ}$ (Figura 21 ).

El movimiento dextrógiro de cada tipo de triángulo sobre los vértices del dodecágono produce en cada caso, una construcción geométrica única y diferente y en el proceso se hace notar las posiciones de los triángulos que

17, 18 y 19. Dibujo de María Luisa López Sardá.
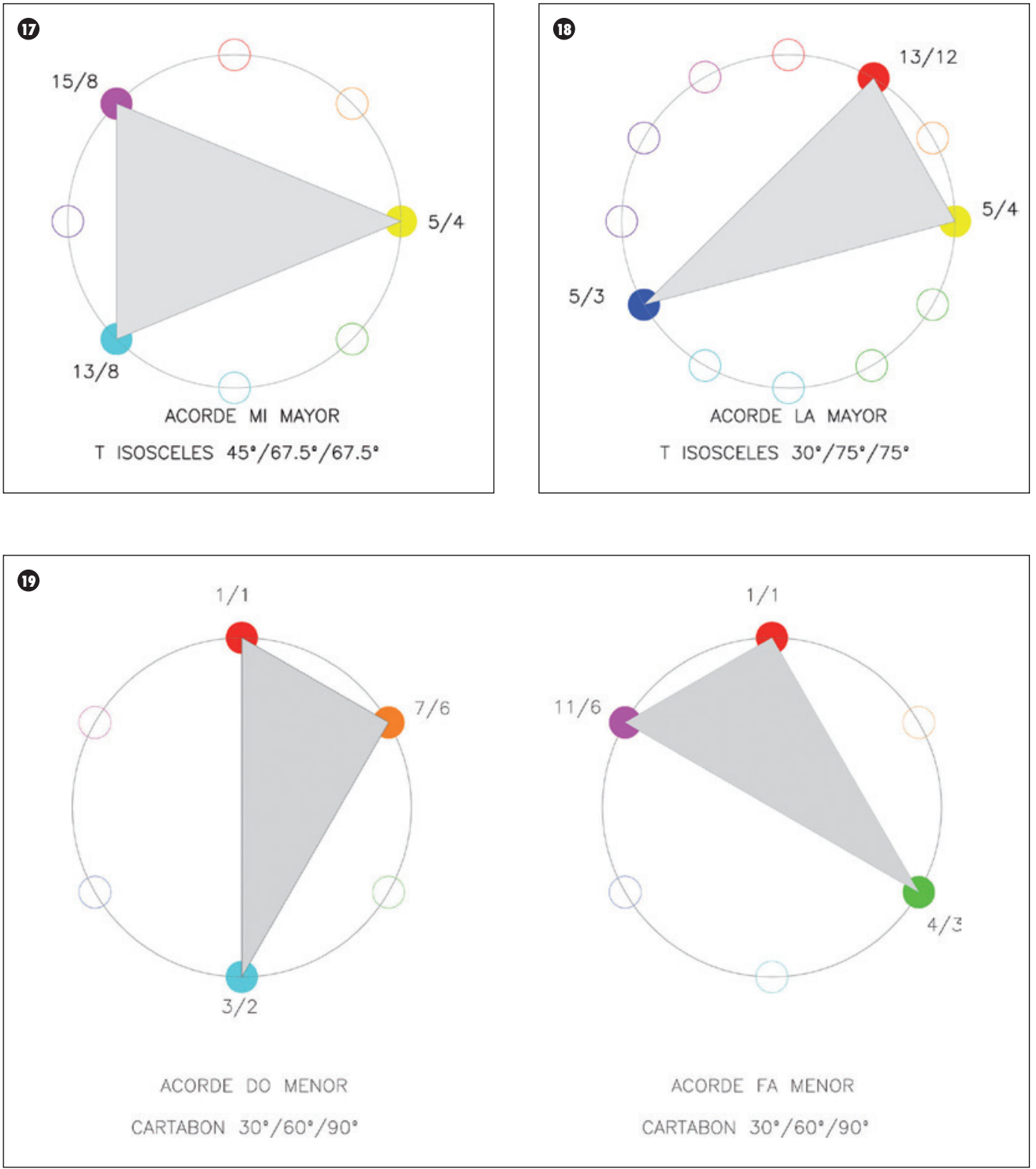

pueden corresponder con acordes musicales (FIgURAs 22 a 26).

No hay que olvidar que estas cristalizaciones no son estáticas. Han sido generadas por el movimiento de cada tipo de triángulo en el tiempo y están inscritas en la circunferencia cuya longitud es la longitud de la cuerda inicial. Si de nuevo, estiramos la cuerda pautada por los nodos o vértices de cada tipo de triángulo, podemos convertir cada posición de cada triángulo en una curva de frecuencia musical y el resultado es una única "forma de onda" de curvatura variable. 
20, 21, 22, 23, 24, 25 y 26. Dibujo de María Luisa López Sardá.
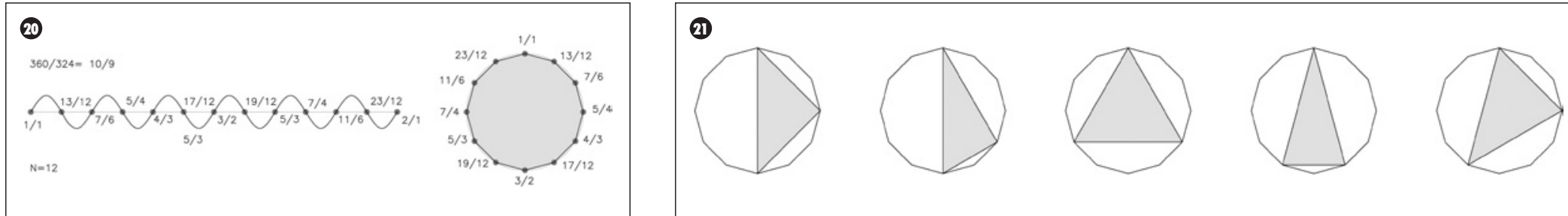

(17)

$$
0 \mathbf{3 0 0 0 0 0 0 0 0}
$$

(3)

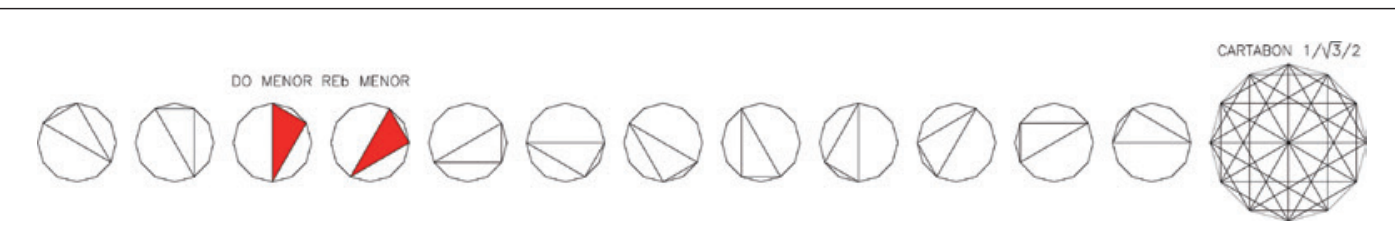

(2)

$$
\text { FA MAYOR FAII MAYOR }
$$

(23)

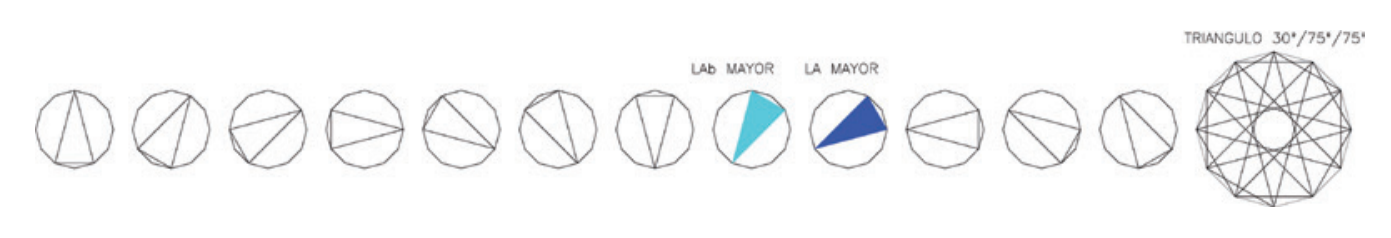

(20)

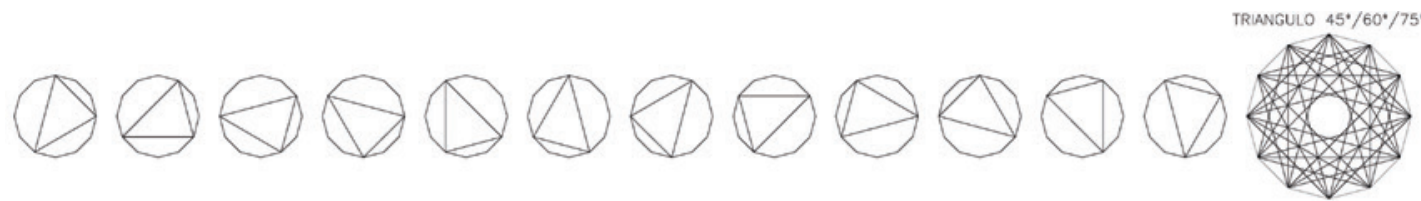

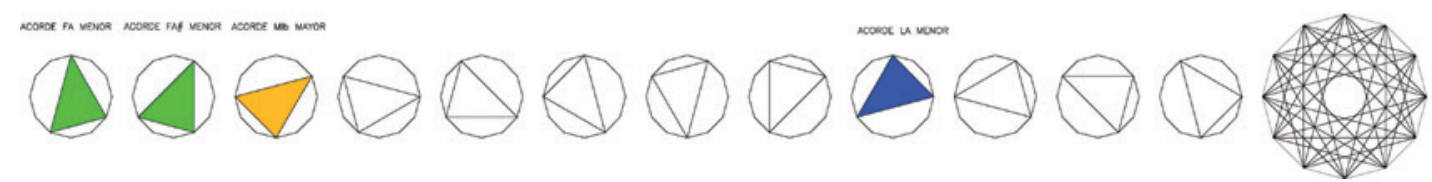


27. Dibujo de María Luisa López Sardá

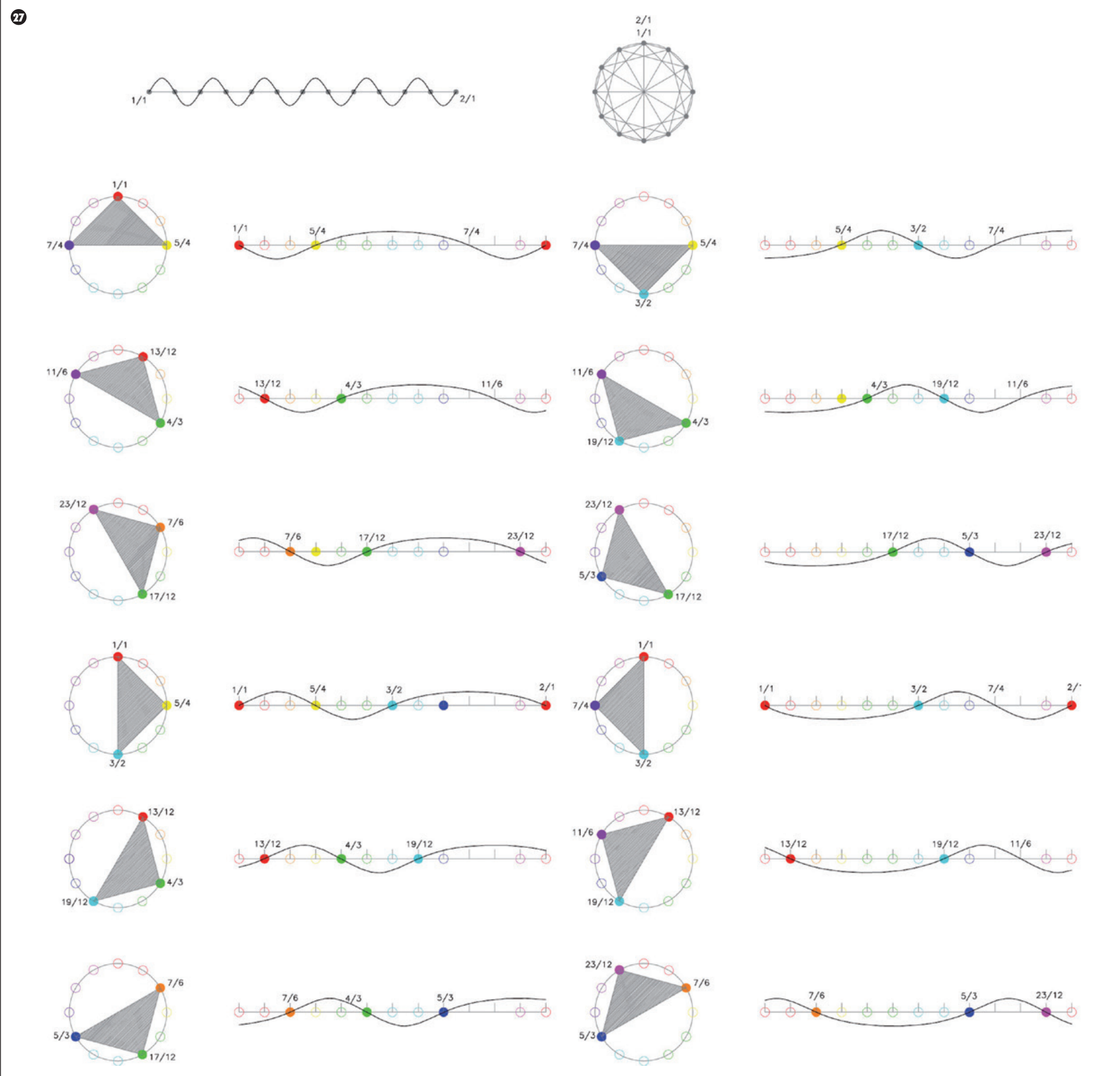


28 y 29. Dibujo de María Luisa López Sardá.

Aunque hemos mencionado las cinco diferentes construcciones geométricas que generan los triángulos constitutivos del dodecágono, todo el desarrollo que vamos a seguir exponiendo estará centrado exclusivamente en el examen de la geometría de la escuadra como ejemplo de esa "otra" geometría de la proporción y la armonía contenida en un triángulo de un polígono regular. Para el estudio se ha tomado el criterio de mantener la amplitud de origen de la cuerda y el sentido dextrógiro de giro.

Situemos el ángulo recto en la posición 1/1 y comencemos el giro sucesivo de la escuadra sobre cada vértice del dodecágono con el ritmo $3 / 6 / 3$. Recogemos la totalidad de las posiciones de la escuadra y sus "formas de onda" y recordemos la dualidad onda-partícula... (FIGURA 27).

Podemos establecer una retícula de la misma longitud de la cuerda espaciada por la distancia entre los nodos e ir colocando las diferentes curvas de frecuencia de la escuadra en su orden correspondiente de generación. Estas retículas son en sí, no dependen de medida al igual que el dibujo geométrico. Son instantáneas congeladas en el tiempo y actúan como semillas que contienen la génesis de cada construcción geométrica. Es una convención que se establece para entender como síntesis esta generación del dodecágono desde el punto de vista ondulatorio. Y por ello, también pueden ordenarse sobre el eje vertical. En ambos casos representan bien en longitud, bien en altura la forma sistemática de vibración del dodecágono según su triángulo característico, en este caso, la escuadra.

En el siguiente dibujo se ha situado a cada lado de la figura geométrica ambas oscilaciones de la cuerda (una como onda incidente y la otra como onda reflejada) y el cuarto dibujo reproduce cada posición de la escuadra como onda estacionaria (FIGURA 28).

Así como podemos superponer dos figuras geométricas manteniendo sus condiciones de simetría, igual podemos hacer con las diferentes

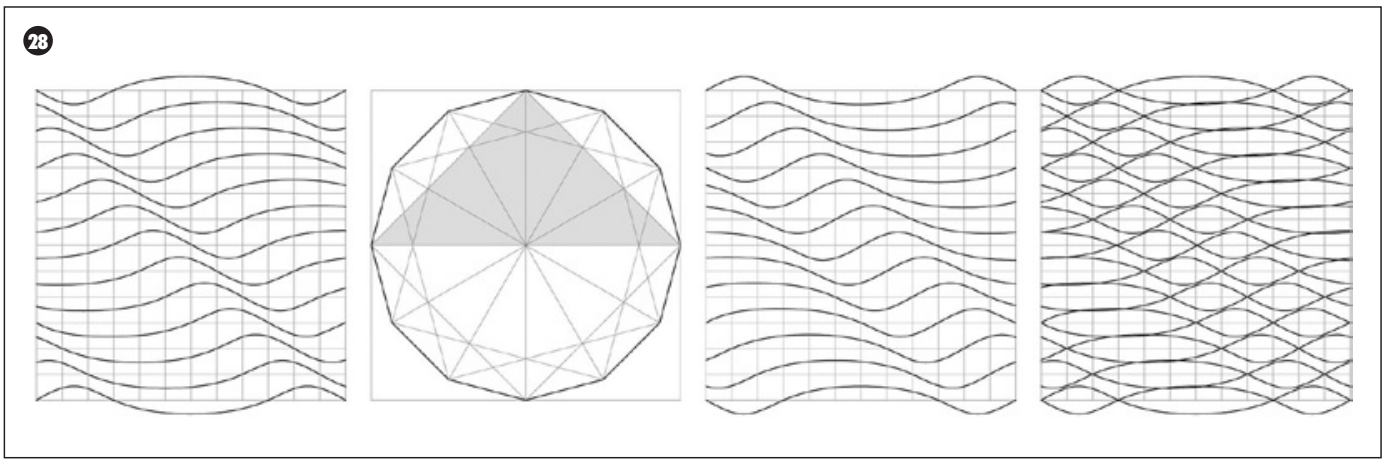

(2)
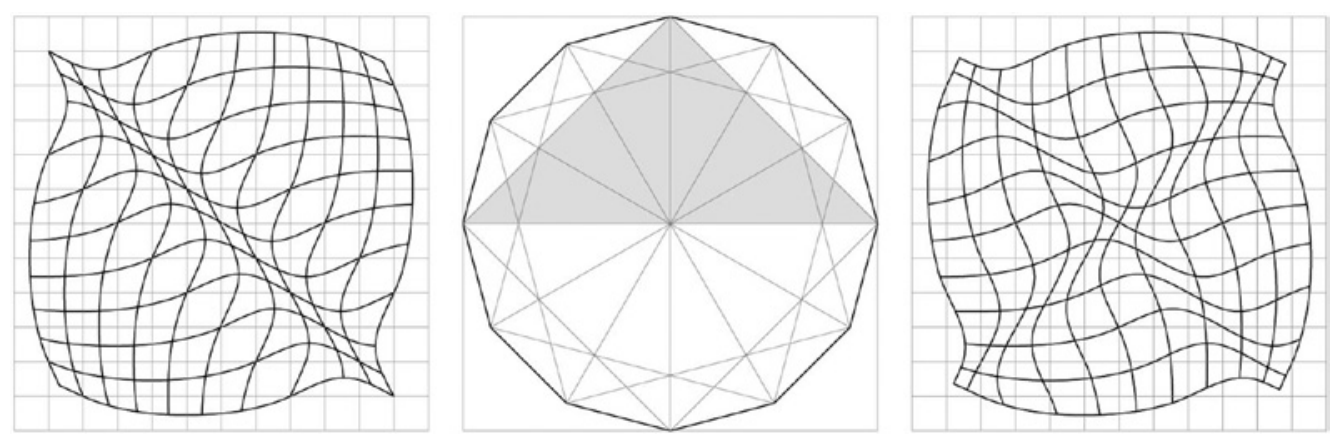

retículas obtenidas de los diferentes triángulos. Si entrecruzamos ambas retículas horizontal y vertical, empezamos a encontrar que no solo la retícula base vibraría en una dimensión o dirección sino que la propia retícula se "arrugaría" formando superficies de valles y crestas dando origen a membranas vibrantes en el tiempo.

Se han utilizado dos criterios de superposición aunque ambos utilizan la ortogonalidad. El primer criterio, representado a la izquierda plantea un giro de $90^{\circ}$ y su espejo. El segundo criterio, representado a la derecha supone un giro de $90^{\circ}$.

Pero lo más importante es que la ortogonalidad de ambos criterios de superposición nos ha introducido desde el mundo del plano al mundo del espacio (FIgura 29).
Tal vez lo más llamativo de la Figura 29 es ver la figura geométrica en el centro, rodeada por dos tipos de membrana, consecuencia de su aspecto ondulatorio y de los criterios de superposición. Esto significa que una de las características esenciales de la geometría, la dimensión o la extensión, es decir, la longitud, anchura y altura, ha sido sustituida por la amplitud y la longitud de onda, lo que implica la introducción del tiempo como generador. Esto es: frecuencia, pulsación, velocidad y sobre todo, energía porque recordemos: una onda es una forma de propagación acompañada de una transmisión de energía...

$Y$ estas semillas-membranas tienen capacidad de extenderse y propagarse...

Esta capacidad de extensión de las membranas con leyes de formación y características propias, 
30 y 31. Fotografía de María Luisa López Sardá.

¿no es acaso el concepto de campo de la mecánica cuántica? ¿̇Un campo no se extiende en una zona mucho más amplia que la que ocupa la partícula?

¿No supone esto un cambio en la percepción del propio objeto, el polígono, hacia un proceso de patrones y modelos organizativos?

Así como en física se considera que una partícula es una expresión manifiesta de un campo, ¿̇podríamos considerar los polígonos regulares (como objetos o partículas) como expresión de patrones o membranas ondulatorias?

¿Acaso la agudeza de los ángulos de un triángulo en su conversión a curvas de frecuencia, no indica mayor frecuencia o velocidad de ondulación y siguiendo a Einstein, en definitiva, en mayor gravedad con lo que supone de mayor arrugamiento del continuo espacio tiempo y por tanto de forma o límite más característico?

Estas fotos están tomadas en una piscina. El volumen del agua casi quieto permite ver el fondo de esa manera. Un ejemplo que permite mostrar que lo que estamos describiendo no está tan lejano de la vida cotidiana... (FIGURAs 30 y 31 ).

Una forma de percibir la movilidad de las membranas, es mediante sus desplazamientos. En este caso, hemos utilizado el segundo criterio de superposición de la semilla-membrana generada por la escuadra (FIgURA 32).

En los dibujos inferiores de la Figura 32 puede verse a la izquierda, la semilla-membrana generada por la escuadra con la posición del ángulo recto en la posición $1 / 1$ y en la parte superior, su extensión horizontal. El primer desplazamiento es un giro de $90^{\circ}$ en la posición del ángulo recto: un desplazamiento de tres nodos y la posición 5/4 para el ángulo recto; en la parte superior, la misma membrana desplazada tres instantes de tiempo. El segundo desplazamiento es un de giro de $270^{\circ}$ en la posición del ángulo recto: un desplazamiento
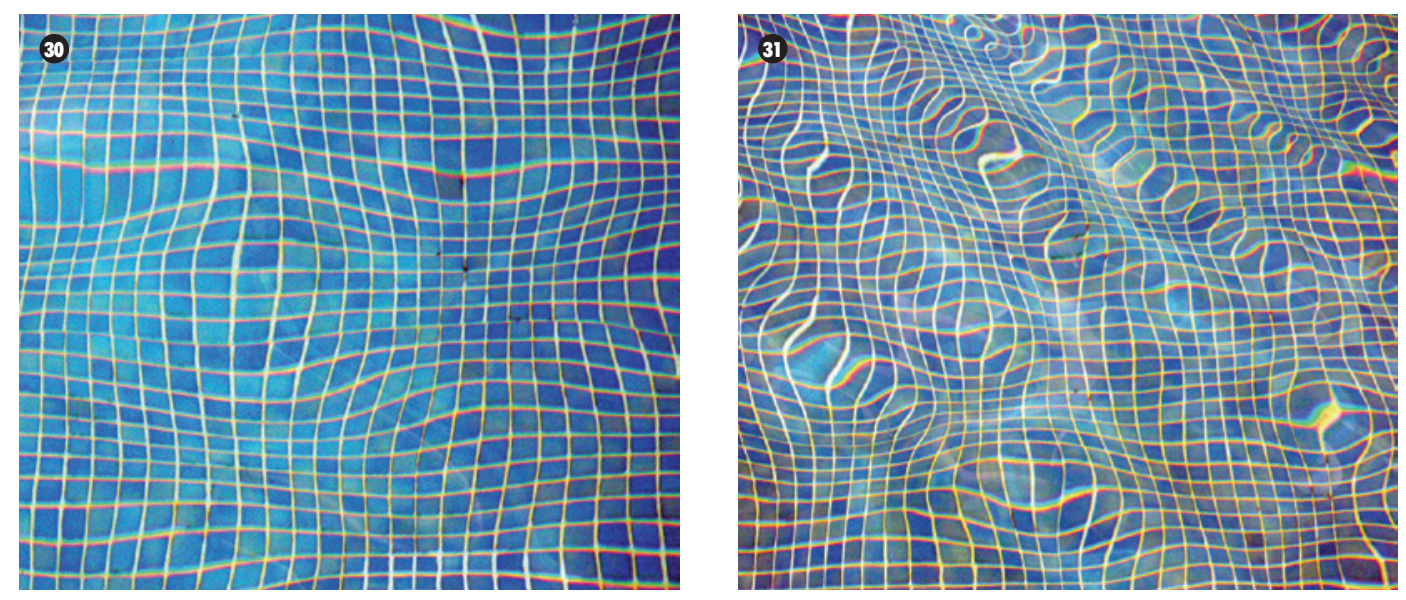

de nueve nodos desde el origen y la posición 7/4 para el ángulo recto. Arriba de nuevo, la misma membrana desplazada nueve instantes de tiempo. Esto es, en la parte superior del dibujo, la misma membrana en tres instantes de tiempo; en la parte inferior: sus tres instantes de comienzo.

O dicho de otro modo, tres energías potenciales de comienzo de la misma membrana.

$Y$ aunque estamos exponiendo el caso de la escuadra, podemos afirmar que los desplazamientos de cada membrana generada por cada triángulo constitutivo del dodecágono ponen en evidencia la importancia del tiempo o la unidad entre espacio y tiempo: el "aquíahora" de cada creación. El "ahora" comienzo define la "forma geométrica" de la membrana o su propia "energía potencial". Esto es, Energía, Forma y Geometría quedan fijadas en el "Ahora" o instante de comienzo...

\section{A. Schönberg (2005), poéticamente, sintetiza este proceso:}

"Y por tanto sucede que en su desarrollo, ningún instante es igual a otro. Cada etapa sirve al mismo tiempo de preparación para la próxima. Es una eterna transformación, un crecimiento ininterrumpido de nuevos brotes de la misma semilla. Está claro entonces por qué dos puntos ampliamente separados en este desarrollo son tan distintos y distantes entre sí. De tal relación como conjunto. Únicamente, después de un estudio más acabado, percibimos en la potencialidad del primer período la certidumbre del segundo".

Antes de seguir, conviene hacer una pequeña recapitulación. Comenzamos planteando la posibilidad de revisar los conceptos de geometría, proporción y armonía a la luz de las nuevas teorías físicas y las nuevas realidades del espacio tiempo que implican.

Y parece que ahora, a comienzos del siglo XXI, todos los adelantos y descubrimientos que desde mediados del siglo XIX y todo el siglo XX, se han ido realizando y que han afectado de lleno a la comprensión del espacio tiempo, no han rozado siquiera a determinados conceptos que la arquitectura utiliza. Al quedarse de alguna manera, "inútiles o rezagados" de la cultura existente, el solo hecho de mencionarlos, puede entenderse como fruto de un interés meramente erudito; han pasado de ser conceptos esenciales a accidentales. Pero lo que estamos planteando sobre proporción y armonía está en la base de estos conceptos, es comenzar por el principio, cuando se definieron los conceptos de proporción y armonía y su instrumento fue el monocordio o sistema de oscilación de una cuerda. manera que, en principio, no reconocemos su 
32. Dibujo de María Luisa López Sardá.

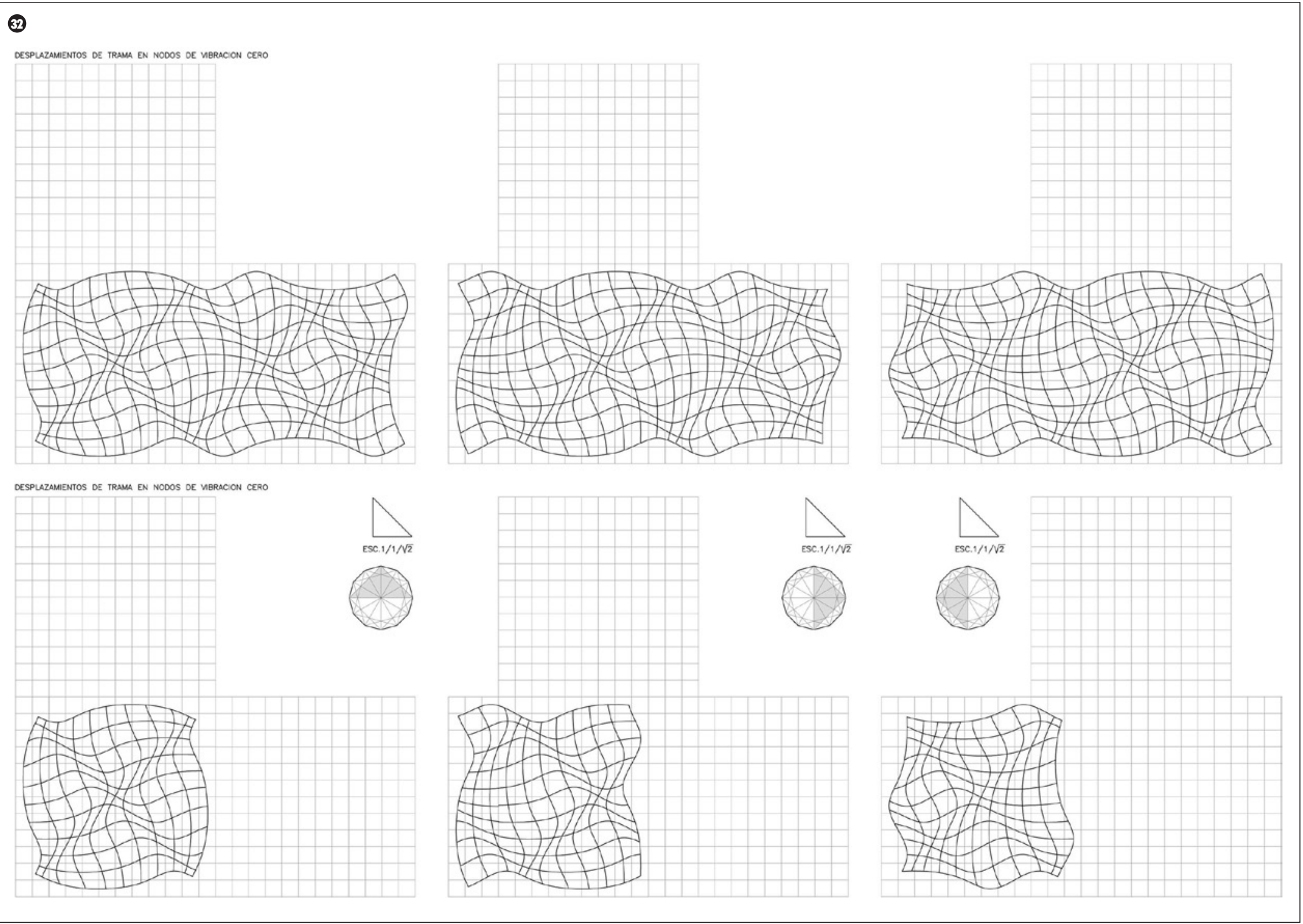

Las etapas que hemos recorrido son:

- Una cuerda, en cualquiera que sea su modo de vibración armónica, puede convertirse en la longitud de una circunferencia. Dicha longitud queda pautada por los nodos del modo de vibración. La unión de estos da origen a un polígono. Este puede descomponerse en sus triángulos constitutivos.
- Cada triángulo constitutivo de cada polígono, genera en su movimiento dextrógiro sobre cada vértice del polígono, una geometría de construcción característica inscrita en la circunferencia cuya longitud define la longitud de la cuerda.

- Esta longitud puede convertirse en tantas cuerdas como número de triángulos contenga el polígono. En cada caso, el modo de vibración de las cuerdas están pautadas por la posición de los vértices de los triángulos, dando tantas "formas de onda" de curvatura variable como posiciones tiene el triángulo.

- Estableciendo una retícula cuyo número de divisiones corresponde al modo de vibración o al número de lados del polígono, podemos ir colocando las diferentes "formas de onda" en su orden correspondiente de generación y el conjunto da origen a un plano ondulado. 
33, 34 y 35. Dibujo de María Luisa López Sardá.

- Estas retículas de "formas de onda" pueden superponerse o entrecruzarse, según criterios establecidos, manteniendo las simetrías básicas de cada polígono y el resultado ha sido una gran variedad de membranas vibrantes.

Dualidad onda-partícula: polígono y retícula ondulatoria. Dos formas de conocer y también hay que decirlo de comportarse: una cosa es lo que conocemos en la geometría euclidiana y otra su forma de vibración. Y tal vez, tendríamos que recordar las palabras de Roger Penrose (2007): ¿̇qué es la realidad? Y añadir, Żes lo que percibimos? o, ¿̇esto es solo el aspecto fenomenológico de la realidad? Aunque tendríamos que aclarar que en las teorías cuántica y de la relatividad general, realmente no existe este dualismo, es más una convención del lenguaje... Según Einstein, la masa no se transforma en energía. Donde hay masa, hay energía. Donde hay partícula, hay campo...

Retomemos las dos semillas-membranas correspondientes a la escuadra según los dos criterios de superposición para estudiarlas según los puntos característicos que definen la amplitud de la onda: la cresta, el nodo y el valle (FIGURAS 33 y 34).

Ambas figuras contienen la construcción geométrica del dodecágono según la escuadra, al lado su retícula de "formas de onda". A continuación la membrana resultante según cada criterio de superposición y por último, las dos matrices de puntos.

Esto es, de la representación del polígono a su representación como retícula de curvas de frecuencia; de la representación de la membrana a su representación como matriz "pixelada" [15]. De la representación en el plano a su representación en el espacio.
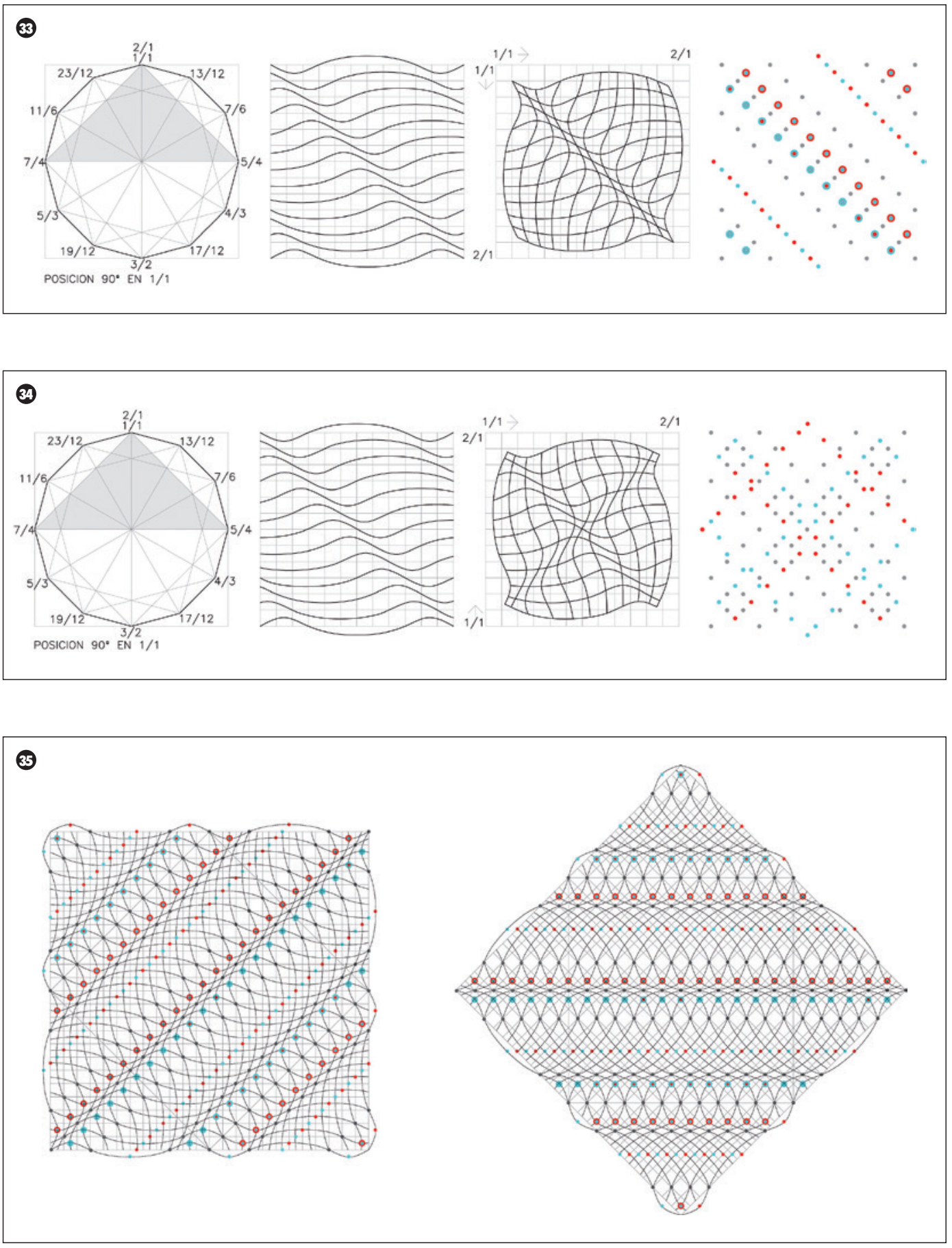

[15] Una imagen digital es una representación de una imagen (que tiene dos dimensiones) como un conjunto finito de elementos pictóricos o píxeles a los que se les asigna, a cada uno, un valor determinado. En términos de percepción, el paso de una entidad continua a una discreta se describe fundamentalmente como un cambio de un ente indivisible y consistente a un objeto completamente segmentado en la que incluso, sus elementos más simples (píxel) pueden percibirse individual y separadamente (Real Jurado, 2005). 
Si tomamos la semilla-membrana de la FIGURA 33, la extendemos, seleccionamos el cuadrado central y lo giramos $45^{\circ}$ y extraemos la membrana resultante y su matriz de puntos, parecería que este giro o este cambio de visión de la misma membrana, nos ha aproximado a una visión más habitual de la arquitectura en su definición del espacio (FIGURA 35).

Esto es, la matriz de puntos establece un orden donde cada punto marca una posición y una condición. Por ello la membrana se ha convertido en un campo: espacio cualificado y definido geométricamente (FIGURA 36).

Podríamos generalizar diciendo:

Si un proyecto es una de las posibles formalizaciones de una idea en el tiempo. Si todas las dimensiones de este proyecto corresponden a un sistema armónico o proporcional de medidas, la geometría del proyecto como estructura profunda de la arquitectura, está necesariamente vinculada a la música y no estaríamos lejos de comprender la afirmación de Goethe (Honour, 1979) cuando decía que la Arquitectura es Música congelada en el Tiempo.

Pero no solo eso, llegados a este punto podemos revisar todo lo planteado. Es decir, ટ̇y si damos la vuelta a todo el proceso? ¿̇Y si las membranas o matrices a las que hemos analizado como un principio o semilla, son realmente los campos en los que los polígonos son solo manifestaciones? O dicho de otra manera, żno son acaso estas membranas patrones y modelos organizativos que llevan intrínsecamente unidos los conceptos de proporción y armonía?

Esto es, en nuestro camino paso a paso, hemos vinculado las proporciones musicales con la geometría, los acordes con los triángulos y este vínculo ha introducido en la geometría, el aspecto temporal de cualquier objeto. Aspecto que ha afectado a la visión de la geometría euclídea, donde el objeto (polígono) se ha convertido en una retícula de curvas de frecuencia y que como tal, puede someterse a procesos de transformación. Pero no solo eso,
36. Dibujo de María Luisa López Sardá.

37. Dibujo de María Luisa López Sardá y Gustavo Jiménez Nieto.

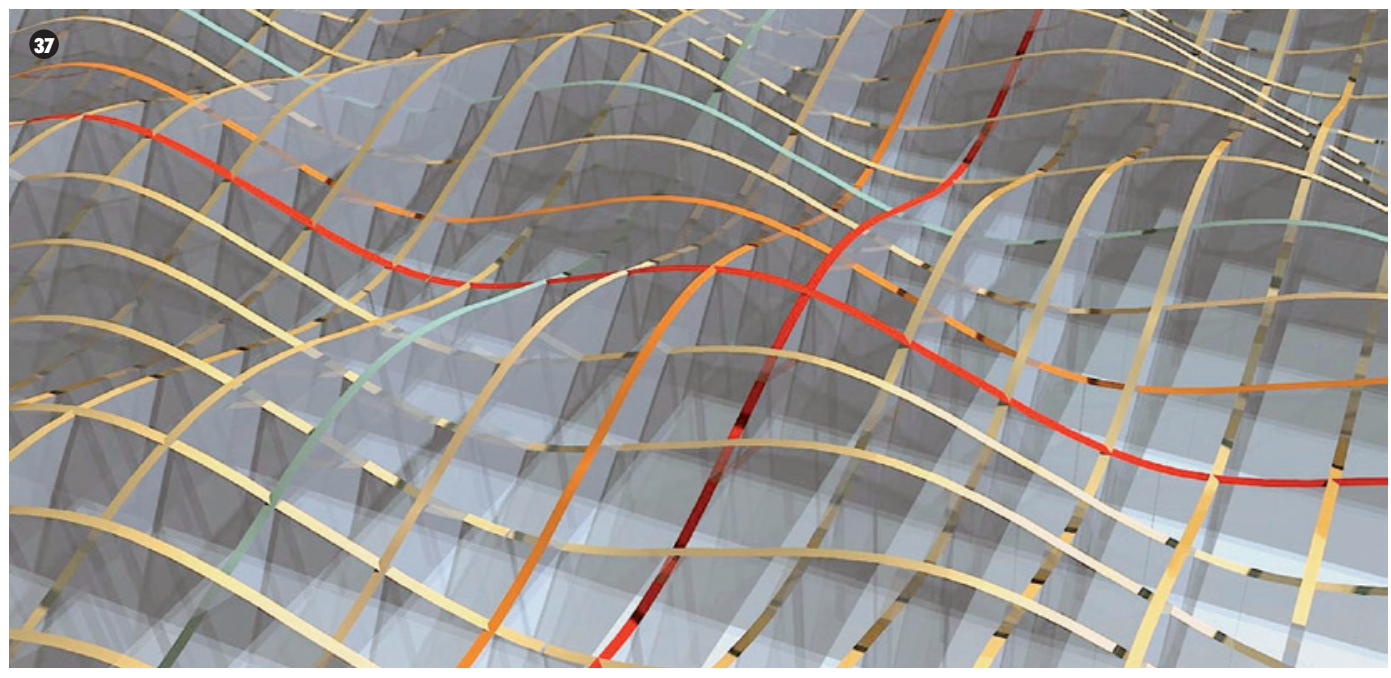

en el propio concepto de onda está contenida su manifestación como propagación de energía y de nuevo, la visión ha cambiado.

Hemos pasado del plano al espacio: al mundo de las membranas que se extienden y propagan. En definitiva, a campos que intrínsecamente están estructurados con las propias leyes de la proporción y la armonía... O dicho de otra manera: la proporción y la armonía han devenido en patrones dinámicos o modelos energéticos de organización del espacio.
Desaparecen los triángulos, los polígonos (objetos o partículas como manifestaciones) y permanecen solo los campos... Campos como entidades con vida propia en los que la tríada de origen: Número, Segmento y Sonido ${ }^{[16]}$ se ha mirado en el espejo y ha reflejado una nueva tríada: Energía, Forma y Geometría que definen y califican a las membranas. Membranas que se han convertido en campos energéticos con leyes y estructura armónica: patrones armónicos y dinámicos de organización del espacio (FIGURA 37).

[16] "Para la visión antigua, el número, el segmento y el sonido se correspondían y eran símbolo de una trascendente unidad" (Borchers, 1970). 


\section{REFERENCIAS}

Borchers, J. (1970). La medición como sustrato del fenómeno arquitectural. En Revista Hogar y Arquitectura $N^{\circ} 87$, marzo-abril. Madrid: Ediciones y publicaciones populares.

Capra, F. El punto crucial. (1992). México: Editorial Pax.

Greene, B. (2007). El Universo Elegante. Barcelona: Editorial Crítica SL. Drakontos Bolsillo
Honour, H. (1979). Romanticism. (1979). New York: Harper \& Row.

Kaku, M. (2008). Universos paralelos. Madrid: Editorial Atalanta.

López Sardá, M. L. (2012). Acordes Arquitectónicos: Otra geometría de la proporción y la armonía en la arquitectura. Madrid: Editorial Fisuras de la cultura contemporánea.

Platón. (1979). Diálogos: Timeo. México: Ediciones Porrúa S.A.
Penrose, R. (2007). El Camino a la Realidad. Barcelona: Random House Mondadori S.A.

Real Jurado, P. (2005). Digitalización de imágenes y topología. En Raúl Ibáñez y Marta Macho (Eds.). Un Paseo por la Geometría. 21 -36. Bilbao: Dpto. Matemáticas. Universidad del País Vasco.

Schönberg, A. (2005). El Estilo y la Idea. Barcelona: Idea Books.

Vitrubio. (1955). Libro I. Cap. II. En Los diez libros de Arquitectura. Madrid: Ediciones lberia. 